D

Dipartimento di Elettronica, Informazione e Bioingegneria, Politecnico di Milano, Milan, Italy.

\section{Physiology of respiratory disturbances in muscular dystrophies}

Muscular dystrophy is a group of inherited myopathies characterised by progressive skeletal muscle wasting, including of the respiratory muscles. Respiratory failure, i.e. when the respiratory system fails in its gas exchange functions, is a common feature in muscular dystrophy, being the main cause of death, and it is a consequence of lung failure, pump failure or a combination of the two. The former is due to recurrent aspiration, the latter to progressive weakness of respiratory muscles and an increase in the load against which they must contract. In fact, both the resistive and elastic components of the work of breathing increase due to airway obstruction and chest wall and lung stiffening, respectively.

The respiratory disturbances in muscular dystrophy are restrictive pulmonary function, hypoventilation, altered thoracoabdominal pattern, hypercapnia, dyspnoea, impaired regulation of breathing, inefficient cough and sleep disordered breathing. They can be present at different rates according to the type of muscular dystrophy and its progression, leading to different onset of each symptom, prognosis and degree of respiratory involvement.

@ ERSpublications

Respiratory failure is a common feature of and the main cause of death in muscular dystrophy http://ow.ly/gyKm305KwZI
Cite as: Lo Mauro A, Aliverti A Physiology of respiratory disturbances in muscular dystrophies. Breathe 2016; 12: 318-327.
According to their aetiology, chronic neurological diseases can be categorised into neuropathic and myopathic disorders. The latter can be acquired or congenital. Muscular dystrophy is a group of congenital myopathies characterised by a variable combination, distribution and severity of 1) progressive skeletal muscle wasting and weakness, 2) defects in muscle proteins, and 3 ) death of muscle cells and tissue [1-3].

Respiratory failure is a common feature in almost all forms of muscular dystrophy, being the main cause of death in these patients, together with heart dysfunction. It is defined as the inability to perform adequately the fundamental functions of respiration: to deliver oxygen (referred to as oxygenation failure) to the blood and to eliminate carbon dioxide from it (referred to as ventilatory failure). The causes of ventilatory failure can involve the respiratory centre, the respiratory muscles or their nerve supply, and abnormalities of the chest wall and pulmonary (lung and airway) systems.

In muscular dystrophy, when the lung is involved, the main cause is recurrent aspiration, as a consequence of either impaired swallowing or episodes of gastro-oesophageal reflux. With the former, aspiration occurs from above when food, saliva, or liquids are breathed into the lungs, whereas with the latter aspiration occurs from below by re-appearance of a bolus of food and/or stomach content into the pharynx. 
The primary manifestation of respiratory failure with lung involvement is hypoxaemia secondary to intrapulmonary shunt resulting from excessive dead ventilation. Hypercarbia may also occur [3].

Muscular dystrophy affects all skeletal muscles. These also include the respiratory muscles, which constitute the respiratory pump, the role of which is to move air into the lung, therefore overcoming the elastic and resistive forces exerted by the chest wall and lungs. The impairment of respiratory muscles (that in muscular dystrophy become progressively weak and/or fatigued), combined with the high load against which the pump has developed its pressure, determine ventilatory failure.

\section{Causes of respiratory load increase}

As shown in figure 1, the elastic properties of the lung are altered in muscular dystrophy patients, with the lung becoming less distensible. The cause of the reduced distensibility of the lung in muscular dystrophy is still not known. Different hypothesis have been proposed: incomplete maturation of lung tissue in case of congenital disease, microor macroatelectasis induced by hypoventilation, increase in alveolar surface tension, fibrosis induced by recurrent aspiration and parenchymal disease. The reasons for the reduction in lung compliance $(C \mathrm{~L})$, therefore, still need to be demonstrated with further dedicated studies. An important factor that, in theory, is capable of reducing lung distensibility is breathing

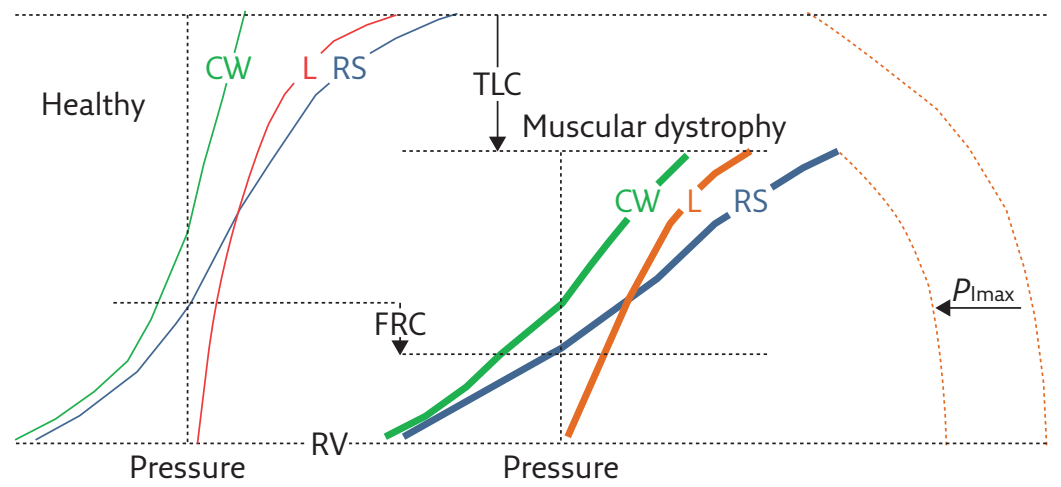

Figure 1 Representative, schematic diagram showing the pressure-volume curves of the respiratory system (RS, blue curves) and its two components, i.e. the chest wall (CW, green curves) and lung (L, red curves), for healthy subjects (thin curves on the left panel) and patients with muscular dystrophy (thick curves on the right panel). The maximal inspiratory pressure (PImax) is also reported (dashed curves). Muscular dystrophy patients are characterised by 1) reduced total lung capacity (TLC); 2) reduced compliance of chest wall (CCW), lungs (CL) and respiratory system (slope of the corresponding pressure-volume curves); 3) reduced PImax; 4) reduced inspiratory capacity $(I C=T L C-F R C)$; and 5$)$ reduced expiratory reserve volume $(E R V=F R C-R V)$. Functional residual capacity (FRC) may be lower or even normal. Residual volume (RV) is not significantly different than in healthy subjects. PImax represents the force of respiratory muscles, while the volume variations are the resulting action of their contraction. The fact that PImax and lung volumes are both reduced indicates that the respiratory muscles, and therefore the respiratory pump, are affected by muscular dystrophy. The compliance of the respiratory system reduces because it decreases in both its components: 1) CL, due to lung atelectasis and fibrosis, the former being a consequence of hypoventilation, induced by respiratory muscles involvement, the latter of recurrent aspiration, induced by the involvement of bulbar muscles; and 2) CCW, due to of the onset of scoliosis, induced by the involvement of trunk muscles. at low lung volume, which is typical in muscular dystrophy. An additional mechanism is the chronic and progressive impairment of respiratory muscles that limits the range of activity of the lung. The elastic properties of a system, in fact, are partly determined by the stress to which the system is subjected. Inspiratory muscle weakness truncates the upper part of the static pressure-volume curve in these patients (figure 1). The position of total lung capacity (TLC), in fact, is the result of the balance between the static elastic recoil pressure of the lung and the pressure generated by inspiratory muscle contraction. The latter is reduced and, therefore, the former is reduced at TLC and this alters the position of the expiratory curve with static expiratory compliance to reduce.

The elastic properties of the chest wall are also altered in muscular dystrophy patients, with the chest wall being stiffer and its compliance (CcW) decreased of about two-thirds of healthy subjects (figure 1). A combination of factors contributes to this: muscle atrophy and osteoporosis, both due to inactivity; extra-articular contractures; progressive degeneration of articular cartilage; and ultimately, kyphoscoliosis often accompanied by chest wall deformity, which is a further mechanical disadvantage for the respiratory muscles. Moreover, since ribcage compliance decreases and abdominal compliance increases in tetraplegic patients, it seems reason to speculate that also in muscular dystrophy patients, the ribcage compartment is the main contributor to the reduction of CCW. The reduced ribcage movements, a consequence of impaired respiratory muscles and decreased physical activity, lead to ankylosis in the costovertebral and costosternal joints, and to stiffening of ribcage tissue, such as tendons and ligaments [2-7].

Upper airway (bulbar) musculature can also be involved in muscular dystrophy patients. The direct consequences are impairment of swallowing, speech impediments and ensuing significant airway obstruction during inspiration [8]

The combination of weakened respiratory muscles with the less compliant chest wall and lungs make pressure swings needed to change lung volume increase and the elastic component of the work of breathing (WOB) increase. Inspiratory airway obstruction makes the resistive component of WOB increase. As a result, both the loads against which the respiratory muscles must act and their WOB increase. With the evolution of the disease, therefore, muscular dystrophy patients have to deal with a progressive imbalance between the load to be overcome and the capacity to overcome it. This is a vicious cycle that predisposes respiratory muscles to fatigue and, ultimately, patients to respiratory failure.

\section{Respiratory muscles}

The diaphragm is the most important inspiratory muscle. External intercostal muscles, sternocleidomastoid and scalene muscles are 
accessory to inspiration, becoming more active in the seated position and during maximal manoeuvres and efforts. The contraction of the inspiratory muscles decreases pleural pressure in order to create a pressure gradient that makes air flow into the lung to ventilate it. As a result, the lung and the ribcage expand. The downward, piston-like movement of the diaphragm increases also intraabdominal pressure, causing an outward movement of the abdominal wall [9-12].

The contraction of the abdominal muscles (transversus, rectus, and internal and external oblique muscles) increases intra-abdominal pressure, making air flow out of the lung and the abdominal wall move inward. When the internal intercostal muscles contract, they exert a large torque that lowers the ribs and increases pleural pressure to drive air outside the lung. Abdominal and internal intercostal muscles are therefore expiratory muscles [13, 14]. At rest, during quiet breathing, expiration is normally passive and driven by the elastic recoil of the lung, whereas during exercise there is an immediate recruitment of abdominal muscles and, to a lesser extent, of the expiratory rib cage muscles [9, 11, 12].

The respiratory pump therefore consists in a coordinated activity of all respiratory muscles to develop the pressures required to drive air in and out of the lung, and to move the ribcage and the abdomen in a coordinated and synchronous way. It is possible to breathe with only one set of respiratory muscles but unwanted effects, like thoracoabdominal paradoxical movement (i.e. one compartment expands while the other deflates), are likely to occur.

- The isolated contraction of the diaphragm inflates the lungs, and makes the abdomen expand and the ribcage move inwardly. This is due to the lack of ribcage muscle tone to counteract the effect of negative pleural pressure swings on the thoracic compartment [9-11].

- The isolated contraction of the accessory inspiratory muscles inflates the lungs, and makes the ribcage expand and the abdomen move inwardly. This is due to the lack of abdominal muscle tone to counteract the effect of negative pleural pressure swings, transmitted through the passive diaphragm, on the abdominal compartment [9, 11, 12].

- The isolated contraction of abdominal muscles deflates the lungs, and makes the abdomen move inwardly and the ribcage expand. This is due to the lack of ribcage muscle tone to counteract the effect of positive abdominal pressure swings, transmitted through the passive diaphragm, on the thoracic compartment [9-11].

Thoracoabdominal asynchrony is an inefficient and costly way to breathe, as part of the respiratory muscle contraction is wasted to distort the chest wall rather than to inflate the lungs [11].

\section{Respiratory muscle weakness, fatigue and strength}

The definition of weakness for a respiratory muscle is the inability to generate adequate levels of pressure and volume in the respiratory cycle, whereas fatigue for respiratory muscles is defined as their inability to sustain a given pressure in response to a constant load.

All muscular dystrophy patients experience respiratory muscle weakness, although to different extents according to the specific form of the disease and to its progression. Sometimes the diagnosis of respiratory muscle weakness is delayed because the daily activity of muscular dystrophy patients is often reduced and their ventilatory demands are therefore limited. For this reason, the onset of respiratory insufficiency can be subtle to the point that most muscular dystrophy patients are not aware that they have lost respiratory muscle strength.

The tension time index relates the force developed by the inspiratory muscles to the time that they are being used and it is proposed as an index of respiratory muscle fatigue. A group of children with neuromuscular disease, including muscular dystrophy, show elevated values of the tension time index. This indicates that the whole inspiratory muscles are prone to fatigue from childhood in some forms of muscular dystrophy [16]. As a consequence, respiratory muscle strength, both for inspiratory and expiratory muscles, is reduced in muscular dystrophy patients [17].

Respiratory muscle failure may occur due to either increased WOB beyond the muscle endurance capacity or weakening of the muscle so that they cannot even sustain the WOB of normal quiet breathing.

The multiple impacts of respiratory muscle pump failure are summarised as follows

- Restrictive pulmonary function Respiratory muscle weakness has a direct (loss of expanding pressure and of elastic recoil) and an indirect (changes in $C L$ and $C \mathrm{CW}$ ) impact on pulmonary function, resulting in reduced TLC, reduced vital capacity (figure 2) and normal or reduced functional residual capacity. The Tiffenau index, i.e. the ratio between forced expiratory volume in $1 \mathrm{~s}$ and forced vital capacity (FVC), may be either normal or even high $[2,15,18]$. The restriction of the chest wall is further worsened by scoliosis, which develops in almost all muscular dystrophy forms in which postural muscles are involved.

- Hypoventilation and thoracoabdominal pattern With the progression of the disease and respiratory muscle involvement, muscular dystrophy patients are more prone to rapid, shallow breathing (RSB) (figure 2), and asynchronous movement between the ribcage and abdomen. RSB is the result of the combination of at least three factors: respiratory 


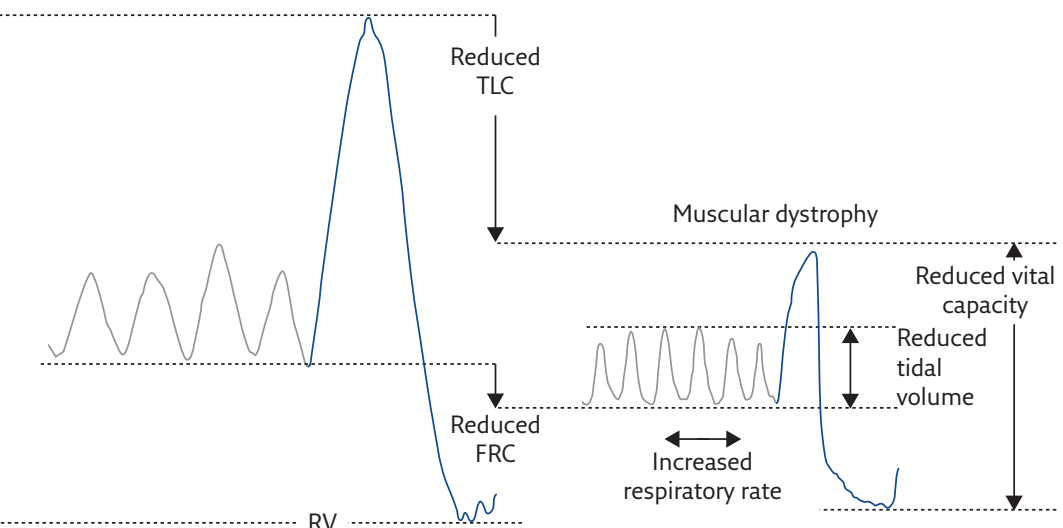

Figure 2 Lung volume variations during quiet breathing (grey lines) and vital capacity (blue lines) in an healthy subject (left) and a muscular dystrophy patient (right). Muscular dystrophy patients are characterised by reduced vital capacity, and rapid and shallow breathing at rest, i.e. reduced tidal volume and increased respiratory rate. RV: residual volume; FRC: functional residual capacity.
- Impaired regulation of breathing Muscular dystrophy is also characterised by altered feedback from the receptors of the respiratory muscles. A degenerative change in the spindle of the muscle, with consequent impairment of reflexes, is also present in muscular dystrophy patients. The main consequence of such feedback disturbance is an impaired control of breathing. A further factor that may alter the breathing pattern in these patients is a reduced mechanoreceptor output from tendons [19-21].

- Cough The essential features of effective cough function are bulbar muscles to keep the glottis completely closed in the compressive phase and co-activation of antagonist respiratory muscles to generate a deep inspiratory volume of cough, and sufficient pressure to compress and expel the gas. Antagonist respiratory muscle co-activation is also needed to counteract the chest wall distortion secondary to the violent muscular activity during cough. The bulbar and respiratory muscle involvement in muscular dystrophy, therefore, makes cough inefficient in these patients. The operating volume is the volume inspired at the end of the inspiratory cough phase. It is shown to be the most important determinant of peak cough flow, as it affects the expiratory muscle length and, therefore, their efficiency of contraction. The combination of inspiratory muscle weakness, which prevents the patient from taking a deep breath, and the decreased $C \mathrm{CW}$, which decreases the available chest wall excursion, limits the operating volume required for an effective cough. When glottis dysfunction is present, adequate pressures to develop the compressive forces for expectoration of airway secretions are not reached. The effectiveness of the cough is further diminished. The expiratory cough phase, when secretions have to be expelled, is limited by 1 ) expiratory muscles weakness; 2 ) a stiffened chest wall that limits expiration; 3) a mechanical disadvantage of the expiratory muscles, because of not being properly stretched to their optimal point of contraction by the restricted operational point; 4) limited passive elastic recoil of the lungs during expiration, because of their limited inflation; and 5) diminished cross-sectional diameter of the airways that are not fully dilated because the lung is not fully inflated. This increases airway resistance during expiration, further limiting cough flow rates [2, 22, 23].

- Sleep disordered breathing (SDB) Upper airway and respiratory muscle weakness both contribute to enhancing the likelihood of SDB in muscular dystrophy patients. Upper airway weakness worsens during sleep and induces airway obstruction, therefore predisposing muscular dystrophy patients to obstructive apnoeas or hypopneas. High body mass index and craniofacial abnormalities (i.e. retrognathia or macroglossia) can predispose to an anatomically 
unfavourable upper airway collapse during sleep, and they may both be characteristic features in muscular dystrophy. Respiratory muscle weakness leads to hypoventilation, due to limited tidal volume, during rapid eye movement (REM) and non-REM sleep. While sleeping, minute ventilation falls because the ventilatory drive decreases. This is a consequence of the reduced chemosensor feedback in response to the rise in carbon dioxide partial pressure and bicarbonate retention. In contrast, upper airway resistance increases because the tone of the pharyngeal dilator muscles decreases. These features further worsen during REM sleep, in which the tone of all the skeletal muscles abruptly falls, with the exception of the extraocular muscles and of the diaphragm. As a result, patients with severe upper airway and respiratory muscle weakness experience a significant drop in oxygen saturation and rise in carbon dioxide tension. Hypoventilation and the consequent abnormalities in gas exchange lead to frequent arousals. On the one hand, frequent arousals are a protective mechanism to minimise blood gas worsening, by restoring tone and activity of respiratory and airway muscles; on the other hand, they reduce sleep time and efficiency, resulting in daytime somnolence and fatigue [24, 25].

The aforementioned generalised causes of respiratory disturbances in muscular dystrophy are summarised in figure 3 . They can be present at different rates according to the type of muscular dystrophy and its progression. The degenerative nature of the disease, in fact, may lead to different onset of each symptom, prognosis and degree of respiratory involvement. For this reason, a brief description of the principal muscular dystrophies, with particular attention to the respiratory implications (where available), will follow.

\section{Becker muscular dystrophy}

Becker muscular dystrophy (BMD) is a late-onset form of X-linked muscular dystrophy characterised by slow progression. Respiratory function and spirometry are almost within normal values. Chest wall volumes and thoracoabdominal volumes are also similar to those of healthy subjects during quiet breathing. In a small percentage of BMD patients, lung restriction occurs but not as severely as the problems seen in Duchenne muscular dystrophy $[2,26]$.

\section{Congenital muscular dystrophy}

Congenital muscular dystrophy is a group of rare muscular dystrophies, with an incidence rate of one in 100000 births, with dystrophic symptoms present at birth or in the first months of life. Respiratory implications are reported in the first decade of life. The worsening of scoliosis in these children starts a vicious cycle of pulmonary restriction, ribcage rigidity and nocturnal hypoventilation. Bulbar involvement is not reported [2, 27, 28].

\section{Distal muscular dystrophy}

Distal muscular dystrophy is uncommon and can either be inherited or a consequence of a sporadic mutation. It is characterised by progressive weakness of distal limb muscles. As myopathic

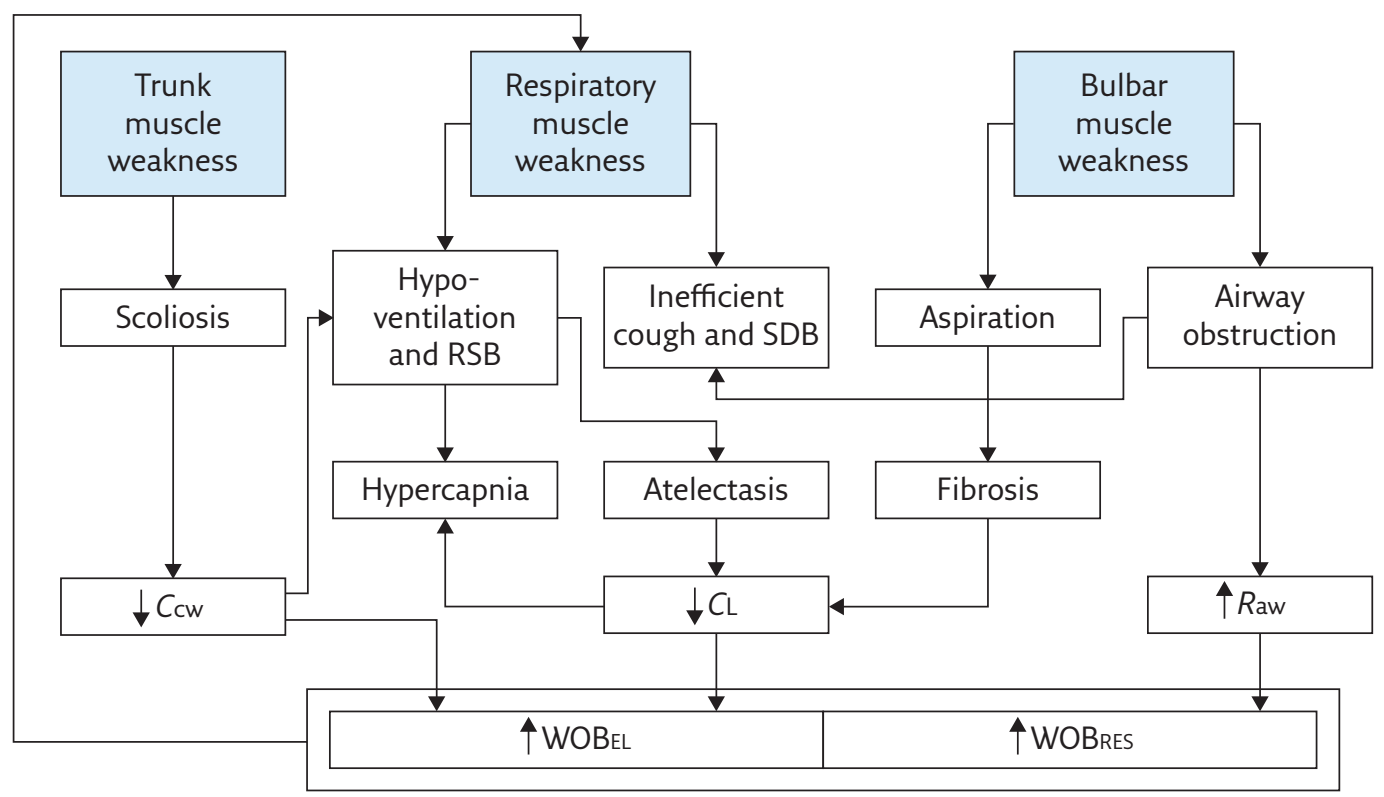

Figure 3 Schematic diagram summarising the generalised causes of respiratory disturbances in muscular dystrophies. Raw: airway resistance; WOBEL: elastic component of work of breathing; WOBRES: resistive component of work of breathing; $\uparrow:$ increment; $\downarrow$ : decrement. 
changes, rather than dystrophic changes, are seen on muscle biopsy, some authors think that it might be more considered as a myopathy rather than a dystrophy. Respiratory involvement is uncommon but recently, a high risk of abnormal respiratory function is shown with progressive worsening in late-onset distal myopathy due to MATR3 mutation $[1,29,30]$.

\section{Duchenne muscular dystrophy}

Duchenne muscular dystrophy (DMD) is the most common inherited progressive myopathy. Having an $\mathrm{X}$-linked recessive inheritance pattern, it affects only males with an incidence of one in 3300 live births. It is caused by progressive lack of dystrophin, a cohesive protein that supports muscle fibre strength, increases muscle stiffness and stabilises the sarcolemma. Its absence is associated with loss of all skeletal muscle function over time. Initially, the proximal lower limb muscles are affected, followed by the shoulder muscles, the distal limb muscles and the respiratory muscles. The consequences are ambulation lost around the age of 10-11 years, progressively worsening scoliosis and breathing difficulties. Without any intervention, death occurs at around 19 years due to cardiac and respiratory failure. With the progression of the disease, patients tend to hypoventilate and increase their RSB index. The breathing rate tends to increase, while tidal volume progressively decreases because of a reduction of the abdominal contribution, particularly in the supine position, which is an index of the action of the diaphragm (figure 4). With the progression of DMD, the diaphragm is characterised by an increased fatigue index, reduced action, paradoxical cephalic movement (in the worst cases) and pseudohypertrophy due to infiltration of connective tissue. The diaphragm in DMD therefore becomes progressively weaker than the other inspiratory muscles and consequently,
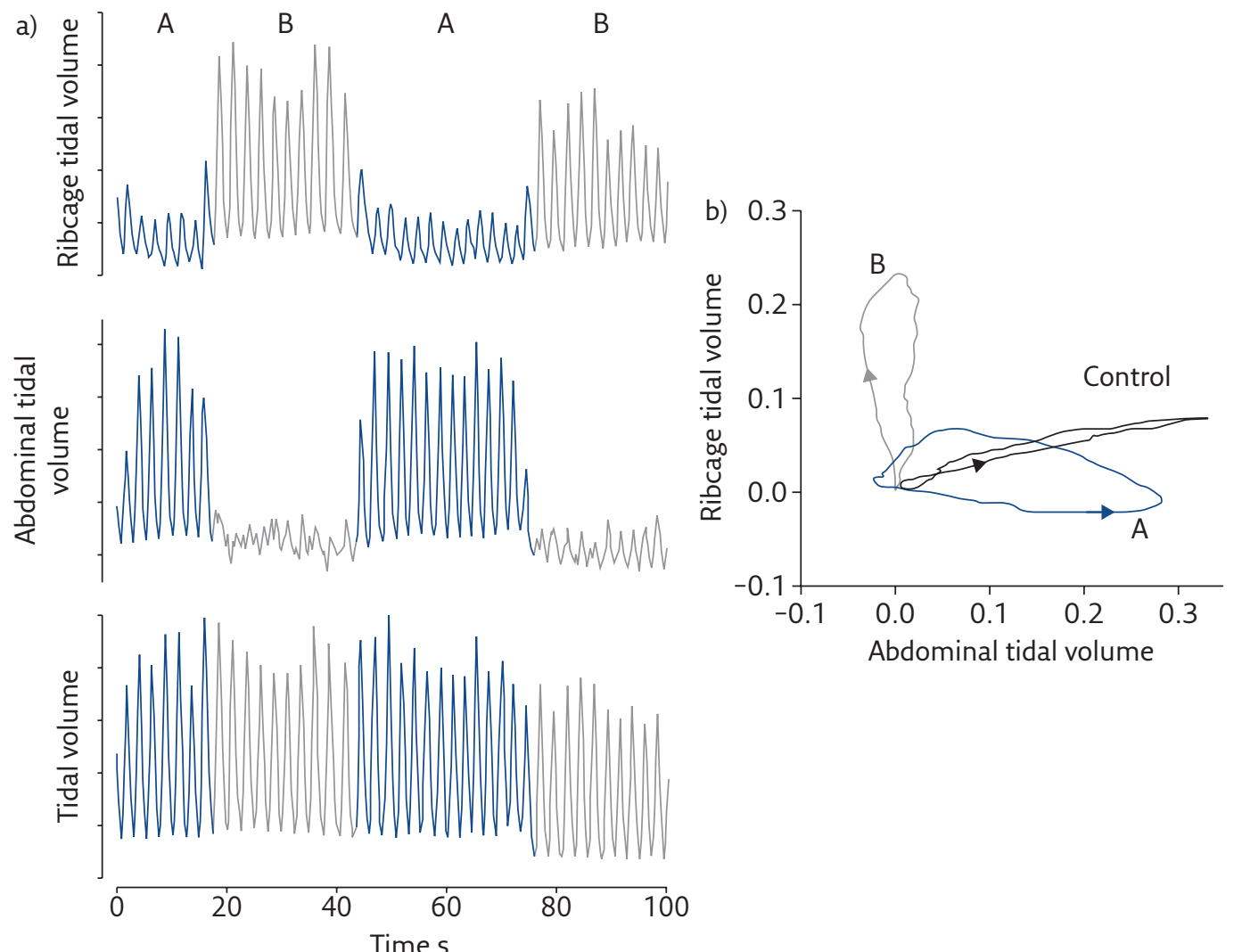

Abdominal tidal volume

Figure 4 a) Ribcage (top), abdominal (middle) and chest wall (bottom) volume variations during spontaneous breathing in supine position in a 77-year-old DMD patient. While tidal volume is constant, the thoracoabdominal pattern alternatively changes by passing from abdominal ( $A$, blue lines) to thoracic ( $B$, grey lines) predominance. $b$ ) "Loops" (i.e. volume changes of the abdomen versus the ribcage) in representative breaths when either the abdomen $(A)$ or the ribcage $(B)$ are prevalent. The breath of a healthy peer (control) is also reported. The arrows indicate the direction of the loops, i.e. the relative action of inspiratory ribcage muscles and the diaphragm [31]. In healthy subjects, both ribcage and abdomen expand during inspiration, with the latter being predominant. This indicates that in the supine position, the diaphragm is the leading muscle of inspiration, with a reduced contribution of ribcage muscles that mainly act to avoid chest wall distortion. The DMD patient therefore alternates periods of breathing in which inspiration is led by the diaphragm $(A)$ and periods in which ribcage muscles are the leading inspiratory muscles (B). These thoracoabdominal "alternans" are thought to be a strategy adopted to cope with diaphragmatic weakness and fatigue. Note that, however, when the diaphragm leads inspiration $(A)$, its action is reduced compared to the healthy subject (i.e. reduced abdominal expansion) and this is an index of diaphragmatic weakness. 
without any intervention, older patients are likely to approach diaphragmatic fatigue.

FVC expressed as percentage of the predicted values linearly declines with age in DMD. Absolute values of all spirometric and plethysmographic parameters follow a pathognomic pattern characterised by ascending, plateau and descending phases. Maximal inspiratory and expiratory pressures are lower in DMD compared to normal values, and decrease with age, with the latter being globally lower than the former. These patients are also characterised by an altered tissue composition of sternocleidomastoid muscle. Taken together, these results indicate that the whole respiratory pump is compromised in DMD, with a global weakness of all respiratory muscles with earlier impairment of the expiratory muscles followed by the inspiratory ones. As a consequence, severe restrictive lung pattern, inefficient cough, severe oxygen desaturation, uncontrolled hypercapnia, nocturnal hypoventilation, SDB and ultimately respiratory failure are expected in older DMD patients [2, 15, 30, 32-43].

\section{Fascioscapulohumeral dystrophy}

Fascioscapulohumeral dystrophy (FSH) is an autosomal dominant dystrophy with a prevalence of one in 20000. Its name is derived from the groups of muscles primarily affected: facial and shoulder girdle; respiratory muscles tend to be spared. In $20 \%$ of FSH patients, trunk muscles, particularly abdominal muscles that lead to a lordotic posture, may be involved as well and this may affect the ventilatory pump function. Facial muscle weakness may complicate spirometric assessment, which can yield less accurate results. Vital capacity and its two components, inspiratory capacity and expiratory reserve volume, are lower in $\mathrm{FSH}$ patients also when assessed by optoelectronic plethysmography, i.e. without the use of a mouthpiece. Maximal mouth pressures measurements may result unreliable. The weakness of upper airway muscles makes these patients prone to obstructive sleep apnoeas [1, 2, 26, 30, 44, 45].

\section{Limb-girdle muscular dystrophy}

Limb-girdle muscular dystrophy (LGMD) is a heterogeneous group of autosomal dominant (types $1 \mathrm{~A}-1 \mathrm{G}$ ) or recessive myopathies (types $2 \mathrm{~A}-2 \mathrm{~L}$ ). The symptoms usually start to be evident around the second or third decades of life. Respiratory involvement is rare in LGMD1 but a restrictive pulmonary pattern may develop in case of severe scoliosis and spinal contracture-induced rigidity. The majority of LGMD2 patients have global respiratory muscle (both inspiratory and expiratory) involvement with significantly reduced contribution of the abdomen, particularly in wheelchair-bound patients. In type $2 \mathrm{~A}$, the involvement of the respiratory muscles occurs late. Type $2 B$ LGMD shows a tendency for respiratory function to decline with disease duration. Types 2C-2F LGMD, or sarcoglycanopathy, show high respiratory morbidity with FVC below $40 \%$ of predicted values. LGMD $2 \mathrm{~L}$ patients develop diaphragmatic weakness, even in ambulant patients, with consequent chronic hypercapnia and nocturnal hypoventilation [1, 26, 30, 44, 46-48].

\section{Emery-Dreifuss muscular dystrophy}

Emery-Dreifuss muscular dystrophy (EDMD) is a genetically heterogeneous dystrophy characterised by a triad of clinical features, namely, early contractures, progressive wasting and weakness of humeroperoneal muscles, and cardiomyopathy being the main cause of morbidity. EDMD patients also develop scoliosis and spinal rigidity that may lead up to a restrictive pulmonary pattern [1, 30].

\section{Educational questions}

1. Regarding muscular dystrophy, which of the following is true?

a) In these patients, total lung capacity and vital capacity reduce, whereas functional residual capacity may be normal or lower.

b) Upper airway musculature is never involved in these patients.

c) Chest wall becomes more elastic with the progression of the disease and therefore its compliance increases.

d) Sleep disordered breathing occurs only in patients with high body mass index and craniofacial abnormalities.

2. A muscular dystrophic patient shows paradoxical thoracoabdominal movements during resting quiet breathing, characterised by outward ribcage movement and inward abdominal movement. The patient is:

a) using the diaphragm to inspire and intercostal muscles to expire.

b) using the diaphragm to inspire with passive expiration.

c) using the intercostal muscles to inspire with passive expiration.

d) using the intercostal muscles to expire with passive inspiration.

3. How does the work of breathing (WOB) change in muscular dystrophy?

a) It does not change because respiratory muscles are not affected by dystrophy.

b) It does not change because although the resistive component of WOB increases, the elastic component of WOB decreases because the chest wall becomes more compliant over time.

c) It increases because airway obstruction increases, and both chest wall and lung compliance decrease over time.

d) It increases because airway obstruction decreases, the chest wall stiffens and the lungs become more elastic over time.

4. Is coughing altered in dystrophic patients?

a) No, because the muscles responsible for coughing are spared.

b) Yes. It becomes more efficient thanks to a combination of physiotherapy and use of cough assisted devices.

c) No, because although the inspiratory cough phase is compromised, the compressive and expiratory cough phases compensate for it.

d) Yes. It becomes less efficient because of inspiratory, bulbar and expiratory muscles weakness that impairs the three cough phases 


\section{Myotonic dystrophy type 1}

With an incidence of one in 8000, myotonic dystrophy type 1 (MD1), or Steinert's disease, is the most common muscular dystrophy in the adult population, with inheritance that occurs in an autosomal dominant manner. With a smaller incidence, congenital and childhood MD1 may also occur. In neonates, the mortality is around $25 \%$ by 18 months of age, with respiratory failure being the main cause, while severe mental impairment remains in those who survive.

MD1 is a multisystem disorder with a typical clinical presentation of bilateral facial weakness, ptosis, muscle wasting, myotonia (i.e. after contraction, the muscle is delayed in its relaxation), cardiac conduction defects and intellectual impairment. Respiratory problems are typical and, with heart involvement, represent the most important cause of morbidity and mortality. Respiratory impairment usually starts in middle age, and it can present different and multiple manifestations: a progressive pulmonary restrictive pattern, irregularity in the breathing pattern (in terms of respiratory rate, tidal volume and fluctuation of resting expiratory levels) whilst awake, diaphragmatic paralysis, respiratory (both expiratory and inspiratory) muscles weakness, SDB, and obstructive or central apnoeas. These patients are also characterised by both abnormal afferent information from muscle spindles and impaired central respiratory pathways. The former seems to be the cause of the highly irregular breathing pattern while awake and the latter of hypercapnia and SDB. The high tendency toward hypercapnia and the early onset of SDB in MD1 is disproportionate, and greater than in nonmyotonic neuromuscular disease for similar levels of respiratory muscles weakness. These patients tend to be overweight, this being an additional factor that strongly affects the severity of nocturnal desaturation. Bulbar weakness can also be present, making MD1 patients prone to respiratory infection, while aspiration of gastric content may occur due to insufficient peristalsis. The cognitive impairment and the altered personality of these patients may hamper the efficacy of noninvasive mechanical ventilation $[2,15,30,46,49-53]$.

\section{Oculopharyngeal muscular dystrophy}

This inherited disorder has an incidence of one in 100000 , and can be either dominant or recessive. The onset of oculopharyngeal muscular dystrophy (OPMD) is around the third decade of life, and it affects extraocular, upper facial, neck, and proximal upper and even lower limb muscles. Typical clinical symptoms are ptosis, ophthalmoplegia and dysphagia. Although respiratory muscles are not involved and early respiratory involvement is very rare, bulbar weakness puts OPMD patients at risk of aspiration pneumonia and obstructive sleep apnoea $[1,30,54,55]$

\section{Suggested answers}

1. $\mathrm{a}$

2. $\mathrm{c}$

3. $\mathrm{C}$

4. d

\section{Conflict of interest}

None declared.

\section{References}

1. Emery AEH. The muscular dystrophies. Lancet 2002; 359 : 687-695.

2. Perrin C, Unterborn JN, Ambrosio CD, et al. Pulmonary complications of chronic neuromuscular diseases and their management. Muscle Nerve 2004; 29: 5-27.

3. Allen J. Pulmonary complications of neuromuscular disease: $A$ Respiratory mechanics perspective. Paediatr Respir Rev 2010; 11: 18-23.

4. De Troyer A. Respiratory effect of the lower rib displacement produced by the diaphragm. J Appl Physiol 2012; 112 : 529-534

5. Criner GJ, Marchetti N. Effects of neuromuscular diseases on ventilation. In: Grippi MA, Elias JA, Fishman JA, et al. eds. Fishman's Pulmonary Diseases and Disorders. 5th Edn. New York, McGraw-Hill Medical, 2015; pp. 1289-1311.

6. Tzelepis GE, McCool FD. Nonmuscular diseases of the chest wall. In: Grippi MA, Elias JA, Fishman JA, et al. eds. Fishman's Pulmonary Diseases and Disorders. 5th Edn. New York, McGraw-Hill Medical, 2015; pp. 1274-1287.

7. De Troyer A, Borenstein S, Cordier R. Analysis of lung volume restriction in patients with respiratory muscle weakness. Thorax 1980; 35: 603-610.

8. Eikermann M, Vogt FM, Herbstreit, F, et al. The predisposition to inspiratory upper airway collapse during partial neuromuscular blockade. Am J Respir Crit Care Med 2007; 175: 9-15.
9. Ratnovsky A, Elad D, Halpern P. Mechanics of respiratory muscles. Respir Physiol Neurobiol 2008; 163: 82-89.

10. Macklem PT. Normal and abnormal function of the diaphragm. Thorax 1981; 36: 161-163.

11. Macklem PT. Respiratory muscles: the vital pump. Chest 1980; 78: 753-758.

12. De Troyer A, Kelly S, Macklem PT, et al. Mechanics of intercostal space and actions of external and internal intercostal muscles. J Clin Invest 1985; 75: 850-857.

13. Kenyon CM, Cala SJ, Yan S, et al. Rib cage mechanics during quiet breathing and exercise in humans. J Appl Physiol 1997; 83: 1242-1255

14. Aliverti A, Cala SJ, Duranti R, et al. Human respiratory muscle actions and control during exercise. J Appl Physiol 1997; 83: 1256-1269.

15. Laghi F, Tobin MJ. Disorders of the respiratory muscles. Am J Respir Crit Care Med 2003; 168: 10-48.

16. Mulreany LT, Weiner DJ, McDonough JM, et al. Noninvasive measurement of the tension-time index in children with neuromuscular disease. J Appl Physiol 2003; 95: 931-937.

17. Misuri G, Lanini B, Gigliotti F, et al. Mechanism of $\mathrm{CO}_{2}$ retention in patients with neuromuscular disease. Chest 2000; 117: 447-453

18. Bourke SC. Respiratory involvement in neuromuscular disease. Clin Med 2014; 14: 72-75. 
19. Swash M, Fox KP. Abnormal intrafusal muscle fibres in myotonic dystrophy: a study using serial sections. J Neurol Neurosurg Psychiatry 1975; 38: 91-99.

20. Swash M, Fox KP. The pathology of the muscle spindle in Duchenne muscular dystrophy. J Neurol Sci 1976; 29: 17-32.

21. Baydur A. Respiratory muscle strength and control of ventilation in patients with neuromuscular disease. Chest 1991; 99: 330-338.

22. Kravitz RM. Airway clearance in Duchenne muscular dystrophy. Pediatrics 2009; 123: Suppl. 4, S231-S235.

23. Smith JA, Aliverti A, Quaranta $M$, et al. Chest wall dynamics during voluntary and induced cough in healthy volunteers. J Physiol 2012; 590: 563-574

24. Bersanini C, Khirani S, Ramirez A, et al. Nocturnal hypoxaemia and hypercapnia in children with neuromuscular disorders. Eur Respir J 2012; 39: 1206-1212.

25. Bye PT, Ellis ER, Issa FG, et al. Respiratory failure and sleep in neuromuscular disease. Thorax 1990; 45: 241-247.

26. D’Angelo MG, Romei M, Lo Mauro A, et al. Respiratory pattern in an adult population of dystrophic patients. J Neurol Sci 2011; 306: 54-61

27. Kang PB, Morrison L, lannaccone ST, et al. Evidencebased guideline summary: evaluation, diagnosis, and management of congenital muscular dystrophy. Neurology 2015; 84: 1369-1378.

28. Yonekawa T, Nishino I. Ullrich congenital muscular dystrophy: clinicopathological features, natural history and pathomechanism(s). J Neurol Neurosurg Psychiatr 2015; 86: 280-287.

29. Kraya T, Schmidt B, Müller T, et al. Impairment of respiratory function in late-onset distal myopathy due to MATR3 mutation. Muscle Nerve 2015; 51: 916-918.

30. Shahrizaila N, Kinnear WJM, Wills AJ. Respiratory involvement in inherited primary muscle conditions. J Neurol Neurosurg Psychiatr 2006; 77: 1108-1115.

31. Agostoni E, Mognoni P. Deformation of the chest wall during breathing efforts. J Appl Physio/ 1966; 21: 1827-1832.

32. Lessa TB, de Abreu DK, Bertassoli BM, et al. Diaphragm: a vital respiratory muscle in mammals. Ann Anat 2016; 205: 122-127.

33. LoMauro A, D’Angelo MG, Aliverti A. Assessment and management of respiratory function in patients with Duchenne muscular dystrophy: current and emerging options. Ther Clin Risk Manag 2015; 11: 1475-1488.

34. Lo Mauro A, D’Angelo MG, Romei M, et al. Abdominal volume contribution to tidal volume as an early indicator of respiratory impairment in Duchenne muscular dystrophy. Eur Respir J 2010; 35: 1118-1125.

35. Toussaint $M$, Soudon $P$, Kinnear W. Effect of noninvasive ventilation on respiratory muscle loading and endurance in patients with Duchenne muscular dystrophy. Thorax 2008; 63: 430-434.

36. Mulreany LT, Weiner DJ, McDonough JM, et al. Noninvasive measurement of the tension-time index in children with neuromuscular disease. J Appl Physiol 2003; 95: 931-937.

37. Lomauro A, Romei M, D’Angelo MG, et al. Determinants of cough efficiency in Duchenne muscular dystrophy. Pediatr Pulmonol 2014; 49: 357-365.

38. Jansen $M$, van Alfen $N$, Nijhuis van der Sanden $\mathrm{MW}$, et al. Quantitative muscle ultrasound is a promising longitudinal follow-up tool in Duchenne muscular dystrophy. Neuromuscul Disord 2012; 22: 306-317.

39. Mavrogeni S. Cardiac and sternocleidomastoid muscle involvement in Duchenne muscular dystrophy. Chest 2005; 127: 143

40. Hahn A, Bach JR, Delaubier A, et al. Clinical implications of maximal respiratory pressure determinations for individuals with Duchenne muscular dystrophy. Arch Phys Med Rehabil 1997; 78: 1-6.

41. Phillips MF, Quinlivan RC, Edwards RH, et al. Changes in spirometry over time as a prognostic marker in patients with duchenne muscular dystrophy. Am J Respir Crit Care Med 2002; 164: 2191-2194

42. Gayraud J, Ramonatxo M, Rivier F, et al. Ventilatory parameters and maximal respiratory pressure changes with age in Duchenne muscular dystrophy patients. Pediatr Pulmonol 2010; 45: 552-559.

43. Tangsrud S, Petersen IL, Lødrup Carlsen KC, et al. Lung function in children with Duchenne's muscular dystrophy. Respir Med 2001; 95: 898-903.

44. Grippi MA, Elias JA, Fishman JA et al. eds. Fishman's Pulmonary Diseases and Disorders. 5th Edn. New York, McGraw-Hill Medical, 2015.

45. Parsons SJ, McMurtrie A, Cooke S, et al. Facioscapulohumeral muscular dystrophy assessment and treatment. Orthop Trauma 2009; 23: 180-185

46. Hutchinson D, Whyte K. Neuromuscular disease and respiratory failure. Pract Neurol 2008; 8: 229-237.

47. Fayssoil A, Ogna A, Chaffaut C, et al. Natural history of cardiac and respiratory involvement, prognosis and predictive factors for long-term survival in adult patients with limb girdle muscular dystrophies type 2C and 2D. PLoS One 2016; 11: e0153095.

48. Takahashi T, Aoki M, Suzuki, N, et al. Clinical features and a mutation with late onset of limb girdle muscular dystrophy 2B. J Neurol Neurosurg Psychiatr 2013; 84: 433-440.

49. Ho G, Cardamone M, Farrar M. Congenital and childhood myotonic dystrophy: Current aspects of disease and future directions. World J Clin Pediatr 2015; 4: 66-80.

50. Bourke SC, Gibson GJ. Sleep and breathing in neuromuscular disease. Eur Respir J 2002; 19: 1194-1201.

51. Romeo V. Myotonic dystrophy type 1 or Steinert's disease. Adv Exp Med Biol 2012; 724: 239-257.

52. Bogaard JM, van der Meché FG, Hendriks I, et al. Pulmonary function and resting breathing pattern in myotonic dystrophy. Lung 1992; 170: 143-153.

53. Gilmartin JJ, Cooper BG, Griffiths CJ, et al. Breathing during sleep in patients with myotonic dystrophy and nonmyotonic respiratory muscle weakness. Q J Med 1991; 78: 21-31.

54. Durmus $\mathrm{H}$, Laval SH, Deymeer, $\mathrm{F}$, et al. Oculopharyngodistal myopathy is a distinct entity: clinical and genetic features of 47 patients. Neurology 2011; 76: 227-235.

55. Witting N, Mensah A, Køber L, et al. Ocular, bulbar, limb, and cardiopulmonary involvement in oculopharyngeal muscular dystrophy. Acta Neurol Scand 2014; 130: 125-130. 